\title{
Differentiating Cystic Liver Lesions: A Review of Imaging Modalities, Diagnosis and Management
}

\author{
Marianna G. Mavilia*1 ${ }^{1}$, Tina Pakala ${ }^{2}$, Marco Molina ${ }^{3}$ and George Y. Wu ${ }^{1}$ \\ ${ }^{1}$ Department of Medicine, Division of Gastroenterology-Hepatology, University of Connecticut Health Center, Farmington, CT, \\ USA; ${ }^{2}$ Department of Medicine, Division of Gastroenterology-Hepatology, New York Presbyterian Hospital, New York, NY, USA; \\ ${ }^{3}$ Department of Radiology, University of Connecticut Health Center, Farmington, CT, USA
}

\begin{abstract}
Hepatic cysts (HCs) are frequently discovered incidentally on abdominal imaging. The prevalence of HCs has been reported as high as $15-18 \%$ in the United States. Although most cysts are benign, some are malignant or premalignant. It is important to diagnose cystic lesions in order to properly manage them. Imaging with conventional ultrasound, computed tomography, magnetic resonance imaging, or contrast-enhanced ultrasound can be used to further characterize and diagnose HCs. Ultrasound is typically the first-line imaging modality, whereas more advanced imaging can help narrow down the specific lesion. Contrast-enhanced ultrasound is a newer modality, recently approved in the United States, which offers noninvasive evaluation in real-time. The first step in diagnosis is stratifying risk by differentiating simple and complex cysts. There are several features that can help identify HCs, including septae, mural consistency, calcifications, and quality of cystic fluid. Simple cysts are mainly congenital cysts, but also occur in polycystic liver disease. Complex cysts include mucinous neoplasms, echinococcal cysts, hemorrhagic cysts, cystic hepatocellular carcinoma and other rare lesions. Treatment is indicated in symptomatic cysts or those suspicious for malignant or premalignant features. Treatment modalities include fenestration, aspiration sclerotherapy, or surgical resection.

Citation of this article: Mavilia MG, Pakala T, Molina M, Wu GY. Differentiating cystic liver lesions: a review of imaging modalities, diagnosis and management. J Clin Transl Hepatol 2018;6(2):208-216. doi: 10.14218/JCTH.2017.00069.
\end{abstract}

\section{Introduction}

Cystic liver disease encompasses a heterogeneous group of fluid-filled lesions within the liver parenchyma. Hepatic cysts (HCs) are most often discovered incidentally on imaging.

Keywords: Hepatic cyst; Simple cyst; Complex cyst; Hepatic imaging; Contrastenhanced ultrasound; Treatment.

Abbreviations: $A D C$, apparent diffusion coefficient; BCA, biliary cystadenoma; $\mathrm{BCAC}$, biliary cystadenocarcinoma; CEUS, contrast enhanced ultrasound; CT, computed tomography; DW-MRI, diffusion-weighted magnetic resonance imaging; $E C$, echinococcal cyst; HC, hepatic cyst; HCC, hepatocellular carcinoma; IVC, inferio vena cava; IDPN, intraductal papillary neoplasm; MRI, magnetic resonance imaging; PCLD, polycystic liver disease; PAIR, puncture, aspiration, injection, respiration; US, ultrasound.

Received: 20 October 2017; Revised: 14 November 2017; Accepted: 20 November 2017

*Correspondence to: Marianna G. Mavilia, Department of Medicine, University of Connecticut Health Center, 263 Farmington Ave, Farmington, CT 06032, USA. Tel: +1-860-679-2509, Fax: +1-860-679-6582, E-mail: mmavilia@uchc.edu
Detection of $\mathrm{HCs}$ is rising, due to vast availability and increased use of abdominal imaging modalities. Over the last decade, ultrasound (US) usage has nearly doubled, computed tomography (CT) usage has tripled, and magnetic resonance imaging (MRI) usage has quadrupled. ${ }^{1}$ This increased detection poses two main challenges: first, distinguishing a lesion as either benign or malignant, and second, choosing an imaging modality that will be diagnostically accurate, cost effective and safe.

HCs can be subdivided into simple and complex cysts, based on characteristics of the lesions. Differentiation is important because it can implicate the need for further diagnostics and treatment. The primary means of differentiation is by imaging. Advanced imaging has made it possible to accurately diagnose HCs without invasive testing, such as biopsy or resection, in some cases. There are several modalities that are useful in characterizing $\mathrm{HCs}$, including conventional US, CT, MRI, and contrast-enhanced ultrasound (CEUS).

In this review, we aim to describe the different types of hepatic cysts and how various imaging modalities are used to evaluate and diagnose them. We will also discuss management strategies for HCs.

\section{Epidemiology}

Prior to the widespread availability of diagnostic imaging, HCs were discovered surgically. A study conducted from 1954 to 1971 found the incidence of HCs to be 17 in 10,000 cases. ${ }^{1,2}$ Currently, the prevalence of HCs has been reported as high as $15-18 \%$ in the United States. ${ }^{1,3}$ Simple cysts are the most common, found in $2.5-18 \%$ of the population. ${ }^{3,4}$ Congenital cysts are more common in females of ages 40-70, whereas acquired cysts (including hydatid, traumatic and inflammatory cysts) occur more commonly in males of ages 30-50. ${ }^{1,2}$ Mortality is generally low; ${ }^{5}$ however, the mortality rate ranges according to etiology of the cyst, and increases when cyst complications develop.

\section{Clinical presentation}

The majority of HCs are asymptomatic. As HCs increase in size, they may become symptomatic. ${ }^{1}$ This occurs in $15-16 \%$ of patients with $\mathrm{HCs}^{6}{ }^{6}$ Symptoms are nonspecific and may include abdominal pain, early satiety, nausea, or vomiting. ${ }^{3}$ Patients may have a palpable mass or hepatomegaly on physical exam. ${ }^{3}$ This is also dependent on the size of the cyst (s). Laboratory testing is typically nondiagnostic. There may be mild elevations in liver enzymes, most commonly with 
elevations in alkaline phosphatase and gamma-glutamyl transferase. ${ }^{3}$ If cystic fluid is collected and analyzed, it may have elevated CA19-9 levels. ${ }^{3}$ This can be associated with elevated serum CA19-9 levels. The current evidence suggests that elevated CA19-9 in cystic fluid does not correlate with malignant lesions. A study by Wang et al. ${ }^{7}$ evaluated 21 cases of mucinous cysts, of which $57 \%$ were CA19-9-positive. Interestingly, there was no significant difference in CA19-9 content between the benign biliary cystadenomas (BCAs) and the malignant biliary cystadenocarcinomas (BCACs). ${ }^{7}$ This was a relatively small study, from which several cases were excluded because CA19-9 was not measured.

A larger sample size may be needed to adequately characterize the relationship between CA19-9 and malignancy in HCs. CA19-9 may be helpful in distinguishing BCAs and BCACs from hydatid cysts or hemorrhagic simple cysts in cases where imaging is equivocal. This has only been shown in case reports and small case series. ${ }^{8-10}$ Conversely, a case report by Yanai et al. ${ }^{11}$ reports elevated CA19-9 in a "simple" HC. In this case, the authors refer to the lesion as a simple cyst, however it is described as having partially enhancing and thickened walls. As we will discuss later, these features are more consistent with a complex cyst. Additionally, the cyst was drained and treated with sclerotherapy but the diagnosis was never confirmed histologically. ${ }^{11}$ Without a confirmed diagnosis, it is difficult to interpret the meaning of an elevated CA19-9 level in this case.

\section{Simple cysts}

Simple HCs are lesions with thin, smooth walls, lined with cuboidal epithelium, which secrete bile-like fluid. ${ }^{1-3}$ Simple cysts range in size from $<1 \mathrm{~cm}$ up to $30 \mathrm{~cm}$ in diameter ${ }^{1}$ and may contain up to 2 septa, unlike complex cysts, which are typically multiseptate. Simple cysts include congenital cysts, biliary hamartomas, Caroli disease and polycystic liver disease (PCLD). The majority of simple cysts are congenital, and form from biliary ducts that do not connect to the biliary system. ${ }^{1,3,12}$ Biliary hamartomas are derived from embryonic bile ducts. ${ }^{12}$ Another rare simple cyst occurs in Caroli disease, which is an autosomal recessive disorder characterized by cavernous ectasia of the bile ducts. ${ }^{12,13}$ However, cysts in Caroli disease have a $7 \%$ chance of developing into cholangiocarcinoma. ${ }^{12}$

PCLD is another cause of simple cysts. PCLD is a genetic condition, which can occur concomitantly with polycystic kidney disease or may be confined to the liver. ${ }^{14}$ The liver becomes enlarged, containing multiple cysts (typically $>20$ ), of varying sizes. PCLD is rare, with prevalence ranging from $0.13 \%$ to $0.9 \%$ in the United States. ${ }^{1,15}$ The mechanism of cyst formation in PCLD is not completely understood. It is thought to be related to retained abnormal bile ductules, which become detached from the biliary tree and progressively dilate, forming cysts (Fig. 1). ${ }^{14}$ An alternative proposed mechanism is a defect in biliary cilia, leading to hyperproliferation of cholangiocytes and generation of cysts (Fig. 2). ${ }^{14}$ These proposed mechanisms are portrayed in Figs. 1 and 2, respectively.

\section{Complex cysts}

Complex cysts are defined by the presence of complex features within a lesion, including septations, mural thickening or nodularity, debris-containing fluid, radiographic enhancement, hemorrhagic or proteinaceous contents. ${ }^{12}$ Complex cysts

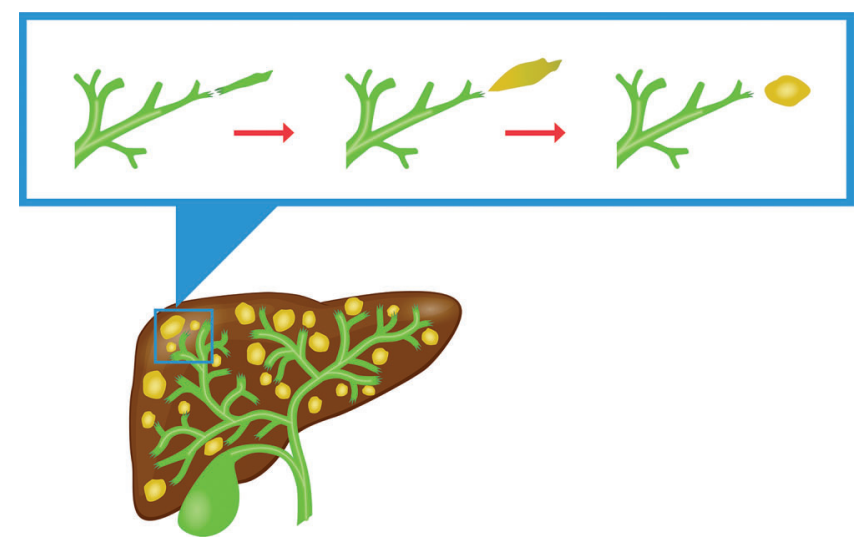

Fig. 1. As the biliary tree is formed, the ducts undergo cycles of apoptosis and regeneration. During this process, small portions of the ductal system may become detached from the main biliary system. These free portions gradually dilate into cystic lesions. ${ }^{14}$

encompass neoplastic, inflammatory, infectious, post-traumatic and other miscellaneous etiologies.

\section{Mucinous cystic neoplasm}

The most common complex cysts are BCAs and BCACs, accounting for $3-5 \%$ of HCs. ${ }^{1,16}$ Together with intraductal papillary neoplasms (IDPNs), BCAs and BCACs constitute a collection of cysts referred to as mucinous cystic neoplasms.

BCAs are multilocular lesions derived from biliary epithelium. ${ }^{3}$ Histologically, they are composed of three layers: a collagenous outer layer; a stromal layer; and, a mucin-secreting columnar epithelial layer. ${ }^{1,15}$ They are slow-growing lesions, ranging in size from $1.5 \mathrm{~cm}$ to $35 \mathrm{~cm} \cdot{ }^{3,16}$ Additionally, they are most commonly found in the right lobe of the liver. ${ }^{12}$ The frequency of malignant transformation of BCA to BCAC is $20-30 \% .{ }^{16,17}$

Differentiation between BCA and BCAC is best done histologically. Multiple studies have proven that imaging is not useful in distinguishing these lesions. ${ }^{13,15,16,18,19}$ Although CEUS is known for its ability to differentiate benign and malignant lesions fairly well, ${ }^{20}$ it has not consistently performed well

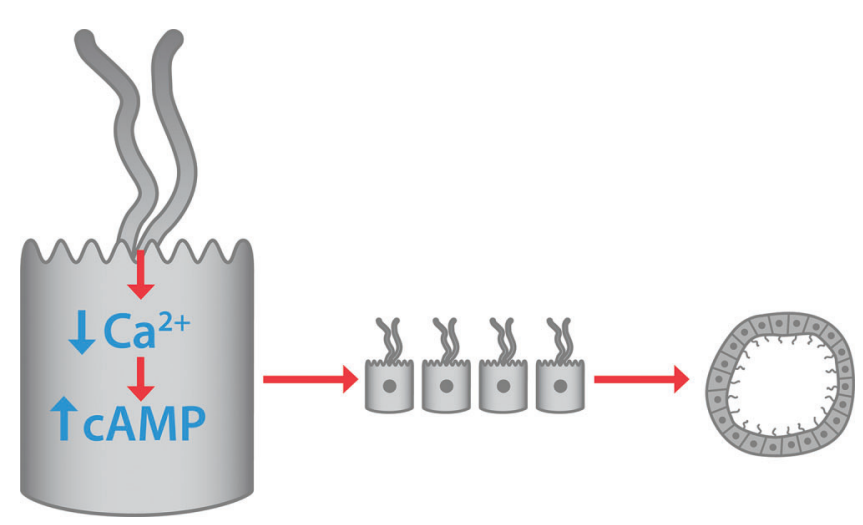

Fig. 2. In a second proposed mechanism of PCLD, the cilia on cholangiocytes detect changes in bile flow or in bile composition, and signal for decreased intracellular calcium and increased levels of cAMP. CAMP then triggers a signaling cascade, stimulating DNA transcription and hyperproliferation of cholangiocytes. The proliferative cells aggregate to form cysts. ${ }^{14}$ 
in distinction between BCA and BCAC. Wall nodularity, particularly nodules greater than $10 \mathrm{~mm}$ in diameter, is more suggestive of BCAC. However, that is not a definitive determinant. ${ }^{13,16,19}$ Several studies have demonstrated the inability of CEUS to distinguish between BCA and BCAC in small studies of $13-36$ patients. ${ }^{16,18,19}$ Despite the small pooled sample of only 72 individuals, the reproducibility among these concordant studies adds reliability to the results.

IDPN is a lesion formed by dilated intrahepatic ducts with intraluminal dysplastic biliary epithelium, which hypersecretes mucous. ${ }^{21,22}$ They are highly differentiated and are considered a precursor to intrahepatic cholangiocarcinoma. ${ }^{21} \mathrm{Kim}$ et al. ${ }^{22}$ conducted a retrospective study comparing imaging and pathologic findings for 62 patients with $\mathrm{HCs}$ in an effort to differentiate mucinous cysts versus simple cysts. They established five findings on $\mathrm{CT}$, which are suggestive of mucinous cyst. These include the presence of septa, central septa, mural nodules, upstream bile duct dilatation, and downstream bile duct dilatation. ${ }^{22}$ This study defined clearly each imaging criteria, which eliminated the often subjective and vague descriptors given by the interpreting radiologist. However, it did not account for interobserver variability, as the analysis was only performed by a single radiologist. Kim et al. ${ }^{22}$ also only evaluated one imaging modality. It would be useful to expand this study design to include US, MRI and CEUS.

\section{Echinococcal cysts}

Hydatid or echinococcal cysts (ECs) occur due to infection with Echinococcus granulosus, a parasite ingested through contaminated food. ${ }^{1}$ Following ingestion, eggs hatch in the small intestine. The parasite then penetrates into the bloodstream, where it can migrate to its target organs-the lungs and liver. ${ }^{1}$ Cysts first appear in the liver 3-4 weeks after infection; ${ }^{1}$ however, they are very slow growing and may exist subclinically for many years. ${ }^{23}$ Pathologically, ECs are fluid-filled with a germinal outer layer. ${ }^{1,3}$ Diagnosis is made in part by clinical history and evidence of parasitic exposure. However, imaging is crucial in making the diagnosis. The sonographic appearance of ECs can be quite variable. Early on, ECs may appear as simple cysts. Over time, they develop a thick and calcified wall with surrounding daughter cysts and several other complex features. ${ }^{1}$

Accurate diagnosis of ECs is important because the mortality of these lesions is slightly higher than for simple cysts, estimated at $2-5 \% .{ }^{1}$ Additionally, ECs can be pressurized, making them prone to rupture with any disruption. ${ }^{1}$

\section{Focal liver lesions that infrequently appear cystic}

Hepatocellular carcinoma (HCC) may present as solid, cystic or mixed lesions in the liver. Areas of necrosis or hemorrhage within the tumor create a cystic appearance on imaging. ${ }^{13}$ HCC may also become cystic after transarterial chemoembolization or radiofrequency ablation. ${ }^{13}$ Following these procedures, necrosis or liquefaction of the lesion lends to a cystic appearance.

In addition to HCC, liver metastases can also appear cystic in certain circumstances. Malignancies that lead to more cystic-appearing liver metastases are neuroendocrine tumors, melanoma, and gastrointestinal stromal tumors. ${ }^{13}$ Cavernous hemangioma is a focal lesion that appears cystic when it has overgrown its blood supply. ${ }^{12}$ Although the above lesions may infrequently appear cystic, they are listed here for completeness in the differential diagnosis of cystic liver lesions.

\section{Miscellaneous complex liver cysts}

Endometrial cysts are very rarely found in the liver, but can mimic the appearance of mucinous cysts. ${ }^{24}$ They are multiloculated with solid and cystic components. ${ }^{24}$ This is an example of extrapelvic endometriosis, occurring when endometrial tissue is implanted in liver parenchyma. ${ }^{25}$ As with pelvic endometriosis, the exact pathophysiology is not completely understood.

Pseudocysts of the liver are posttraumatic cysts that can form in the wake of an intrahepatic hematoma. ${ }^{26}$ There is one case report in the literature of a pseudocyst forming from iatrogenic trauma, as a complication of liver biopsy. ${ }^{26}$

A biloma is a collection of bile that forms a cyst outside of the biliary tract. It typically occurs in the setting of trauma or recent surgery. ${ }^{12}$ Bilomas can present as either simple or complex cysts.

\section{Conventional imaging modalities}

Conventional grayscale US is frequently the first-line imaging modality for screening. Conventional US is widely available, inexpensive, and lacks harmful radiation exposure. ${ }^{27}$ The sensitivity and specificity for US in diagnosis of HCs is about $90 \% .^{3}$ US is able to detect a wide array of lesions, including benign cysts, tumors, and abscesses. However, its role in the differentiation between lesions is limited. Without the ability to evaluate for enhancement patterns, many different types of liver lesions may appear similar on US.

CT has a sensitivity of greater than $90 \%$ for $\mathrm{HCs}^{3,23,27}$ and gives more detailed information about gas contents and calcification within the cyst. ${ }^{27}$ Multiphasic CT imaging uses contrast enhancement and multiple timed acquisitions to yield additional information about liver lesions. ${ }^{28,29}$ Unenhanced or noncontrast imaging is less sensitive for detection of liver lesions. ${ }^{29}$

Several studies have investigated the use of diffusionweighted MRI (DW-MRI) in diagnosis of HCs. DW-MRI is used to calculate the apparent diffusion coefficient (ADC) for a given lesion. This value can then be compared against cut-off values to differentiate the type of lesions. Erturk et al. ${ }^{30}$ conducted a retrospective review of 66 patients with liver metastases, simple cysts or hemangiomas diagnosed by CT or US. After undergoing DW-MRI, the ADC was able to discriminate between these three lesions with high sensitivity and specificity. ${ }^{30}$ Unfortunately, this study relied on CT or US, rather than the gold standard of pathology for confirmatory diagnosis. It also only differentiated between three types of liver lesions. It is unclear how this modality would perform in diagnosis of other types of lesions. In contrast, two similar studies used ADC to differentiate between simple cysts and ECs, in cases where diagnosis was confirmed pathologically. ${ }^{4,31}$ These studies had conflicting results. Inan et al. ${ }^{4}$ demonstrated a difference in ADC values between simple cysts and ECs, whereas Oruc et al. ${ }^{31}$ did not achieve statistical significance in this comparison. Based on this conflicting data, DW-MRI does not seem reliable for definitive diagnosis of HCs.

Table 4 compares and contrasts the strengths and limitations of each imaging modality, which may guide in the selection of a diagnostic study.

\section{An emerging imaging modality}

CEUS is an emerging technique in liver imaging in the United States, but has been well established in Europe and Asia for over a decade. It allows minimally invasive contrast-specific 
Table 1. Distinguishing characteristics of simple versus complex cysts

\begin{tabular}{ll}
\hline Simple & Complex \\
\hline Thin, smooth walls & Septated \\
May contain up to & Mural irregularity or nodularity \\
two septae & Debris \\
& Calcification \\
& Fluid levels \\
\hline
\end{tabular}

imaging and enables operators to visualize and analyze dynamic enhancement patterns in real time. It is recommended by the European Federation of Societies for Ultrasound in Medicine and Biology for diagnosis of liver lesions that are not well characterized by conventional US. ${ }^{18}$

This modality uses a contrast agent, specifically a microbubble agent surrounded by an outer stabilizing shell that is administered intravenously. ${ }^{32}$ Like multiphasic CT, a contrastenhanced liver study is comprised of three dynamic phasesarterial, portal venous and late phases. During each phase, clinicians look for typical vascularization patterns within focal liver lesions. Within the differential diagnosis for focal liver lesions, each type of lesion is associated with a characteristic vascularization pattern. ${ }^{20,32,33}$ In the case of simple liver cysts, there is typically no enhancement on CEUS. However, complex cysts often have enhancing features, which further characterize the lesion and may be diagnostic.

Washout differentiates a lesion from surrounding normal liver tissue and generates information on the lesion's characteristics. It can help identify a $\mathrm{HC}$ as benign or malignant. ${ }^{34}$ Marked hypoenhancement of the lesion during the washout phase may be indicative of malignancy. ${ }^{34}$ However, this feature alone is not always clearly present. Conversely, if there is iso- or hyperenhancement of the lesion during the portal or late phase, this suggests a benign lesion. ${ }^{33,35}$ Additionally, the timing of washout can further characterize the lesion. For example, metastatic lesions have a rapid washout, whereas HCC often has a slow or incomplete washout phase. ${ }^{33,35}$ Of note, the washout pattern in HCC and metastasis demonstrates the opposite behavior on contrast-enhanced CT and MRI. Studies have shown that in $95 \%$ of cases, lesions are able to be distinguished as either benign or malignant during this late phase. ${ }^{20,28}$

In April 2016, Lumason $®$ became the first contrast agent approved by the Food and Drug Administration for use in US of liver in the United States. Definity $\AA$ and Sonazoid $(\AA$ are other agents that have not yet earned this distinction. The large trials that have validated the diagnostic accuracy of CEUS employed clinicians with at least 2-5 years of experience with CEUS. ${ }^{33,36}$
Several studies have shown improved detection of $\mathrm{HC}$ with CEUS, as compared to conventional B-mode US, ${ }^{37,38}$ with detection rates of $85-95 \%$ versus $60 \%$, respectively. ${ }^{37}$ In addition to identifying these lesions, CEUS enables users to characterize and diagnose focal liver lesions in many cases. Two large prospective multicenter studies have validated CEUS as a reliable method for the diagnosis of focal liver lesions. A trial performed by the German Society of Ultrasound in 2008 compared CEUS to liver biopsy. ${ }^{33}$ In that study, 1349 patients from 14 different centers with hepatic tumors seen on conventional B-mode US were examined with CEUS. The lesions were characterized according to enhancement pattern and final diagnosis was compared with diagnosis obtained by histological examination after biopsy of the lesions. The accuracy of CEUS was $90.3 \%$. CEUS was shown to have a sensitivity of $95.8 \%$, a specificity of $83.1 \%$, a positive predictive value of $95.4 \%$ and negative predictive value of $95.7 \%$ for the differentiation of benign versus malignant lesions. ${ }^{33} \mathrm{~A}$ limitation to this study was the lack of blinding to clinical background on each patient, which may have confounded the operator's interpretation of the imaging.

A second trial performed by the French Society of Ultrasound in 2008, compared CEUS to contrast CT or MRI and/or liver biopsy. ${ }^{36}$ At total of 874 patients with hepatic nodules across 15 different centers were included. That study reported sensitivity of $79.4 \%$ and specificity of $88.1 \% .{ }^{36}$ Unlike the prior study, however, imaging was interpreted by two blinded radiologists, in addition to the radiologist performing the sonographic exam. The confirmation by blinded physicians adds to the validity of this trial. Overall, both studies showed good performance of CEUS for the characterization of focal liver lesions, with accuracy ranging from $80 \%$ to $95 \% .^{33,36}$ It is important to note that these two large trials included all focal liver lesions, without specification of solid versus cystic lesions. Therefore, these data may not apply to cystic lesions alone.

\section{Imaging findings}

Imaging findings are unique to each cyst type and can be used to differentiate simple and complex liver cysts. Table 1 details specific imaging features that help distinguish simple from complex cysts. Additionally, findings may differ by imaging modality, with certain modalities more suited for visualized selected features. For instance, calcification is not clearly seen on MRI but is well characterized on CT and can be seen on US. Table 2 details the specific findings for simple versus complex cysts with each imaging modality. In many cases, certain imaging findings can specifically diagnose HCs (Table 3).

Table 2. Appearance of simple versus complex hepatic cysts with various imaging modalities

\begin{tabular}{|c|c|c|c|c|}
\hline & US & $\mathrm{CT}$ & MRI & CEUS \\
\hline $\begin{array}{l}\text { Simple } \\
\text { cyst }\end{array}$ & $\begin{array}{l}\text { Anechoic, homogeneous, } \\
\text { aseptate, thin and smooth } \\
\text { margins }^{1,3,6}\end{array}$ & $\begin{array}{l}\text { Nonenhancing, hypodense, } \\
\text { smooth margins }^{1,3}\end{array}$ & $\begin{array}{l}\text { Nonenhancing } \\
\text { T1: low signal } \\
\text { T2: high signal } \\
3,12\end{array}$ & Nonenhancing ${ }^{6}$ \\
\hline $\begin{array}{l}\text { Complex } \\
\text { cyst }\end{array}$ & $\begin{array}{l}\text { Irregular border, } \\
\text { hyperechogenic } \\
\text { septations, loculations, } \\
\text { shadowing beyond } \\
\text { calcifications }^{1,3}\end{array}$ & $\begin{array}{l}\text { Multilocular, mural and septal } \\
\text { enhancement, mural thickening } \\
\text { and/or nodules, calcifications, } \\
\text { debris containing fluid }{ }^{12,15}\end{array}$ & $\begin{array}{l}\text { T1: hypointense cyst } \\
\text { contents } \\
\text { T2: hyperintense with low } \\
\text { signal border }{ }^{1,23}\end{array}$ & $\begin{array}{l}\text { Mural and septal } \\
\text { enhancement }^{6}\end{array}$ \\
\hline
\end{tabular}




\begin{tabular}{|c|c|c|}
\hline Lesion & Specific imaging findings & Appearance \\
\hline PCLD & All modalities: Multiple cysts & \\
\hline Mucinous cysts & All modalities: Mural or septal nodules & \\
\hline $\mathrm{EC}$ & $\begin{array}{l}\text { All modalities: Thick, calcified laminar wall, floating inclusions, } \\
\text { peripheral daughter cysts }\end{array}$ & \\
\hline Hemorrhagic cyst & $\begin{array}{l}\text { CT/US: Irregularly thickened wall with "flame-like" } \\
\text { prominences }{ }^{10,12} \\
\text { MRI: T1 hyperintensity of hemorrhagic components } 3,12,40\end{array}$ & \\
\hline Cystic HCC & $\begin{array}{l}\text { All modalities: Irregular, may have solid portion } \\
\text { CEUS: Heterogeneous hyperenhancement during arterial } \\
\text { phase, hypoenhancement during portal-late phase, } \\
\text { peripheral rim enhancement }{ }^{13}\end{array}$ & \\
\hline
\end{tabular}

Abbreviations: EC, echinococcal cyst; HCC, hepatocellular carcinoma; PCLD, polycystic liver disease.

On US, simple cysts are anechoic, sharply demarcated lesions demonstrating increased trough transmission ${ }^{1,3}$ (Fig. 3). CT demonstrates well-defined, smooth, nonenhancing hypodense lesions. ${ }^{1,3}$ Fluid-filled center is -10 to 10 Hounsfield units. ${ }^{12}$ The difference in appearance on US compared to CT is demonstrated in Fig. 4. On T1-weighted MRI, simple cysts appear homogeneous with low signal intensity, whereas T2 weighting shows high signal intensity (Fig. 5). ${ }^{1,3,12}$ As with $\mathrm{CT}$, simple cysts are nonenhancing on MRI. Simple cysts are nonenhancing on CEUS in all vascular phases. ${ }^{18}$

Complex cysts show different patterns on imaging. On US, these lesions are hypo-echogenic with irregular walls. Septations can be seen sonographically and there may be shadowing beyond areas of calcification. ${ }^{1,3}$ CT allows better visualization of any calcifications. It is also helpful in distinguishing hemorrhagic contents from simple cystic fluid. ${ }^{15}$ With contrast $\mathrm{CT}$, enhancement may be seen along cyst walls or septae. ${ }^{15}$ CEUS is important for identifying malignant or premalignant complex cysts. They show septal and nodular enhancement in the arterial phase, followed by hypoenhancement in the portal phase. ${ }^{18}$
The typical findings in Tables 2 and 3 serve as a guide to diagnosis. However, there are several cases in the literature which are exceptions to the typical imaging patterns. Watanabe et $a .^{21}$ presented a case of a 59 -year-old male with a large benign-appearing cyst, which was classified on imaging as a simple cyst, lacking septations, mural thickening or any other complex features. It was resected and pathology revealed an oncocytic BCA. ${ }^{21}$ Another case published by Berdel et al. ${ }^{39}$ presented the opposite scenario of simple congenital cyst masquerading as a complex cyst. This patient was a 62-yearold female with a large, septated cyst, containing a fluid level and rim enhancement on imaging. However, pathology revealed a simple biliary cyst. ${ }^{39}$ These instances demonstrate that diagnosis should always be confirmed by the gold standard until more reliable diagnostic imaging is developed.

\section{Pathologic diagnosis}

While pathologic diagnosis by liver biopsy is regarded as the gold standard for diagnosis, it is not without limitations. Histologic evaluation by a pathologist has a level of subjectivity 


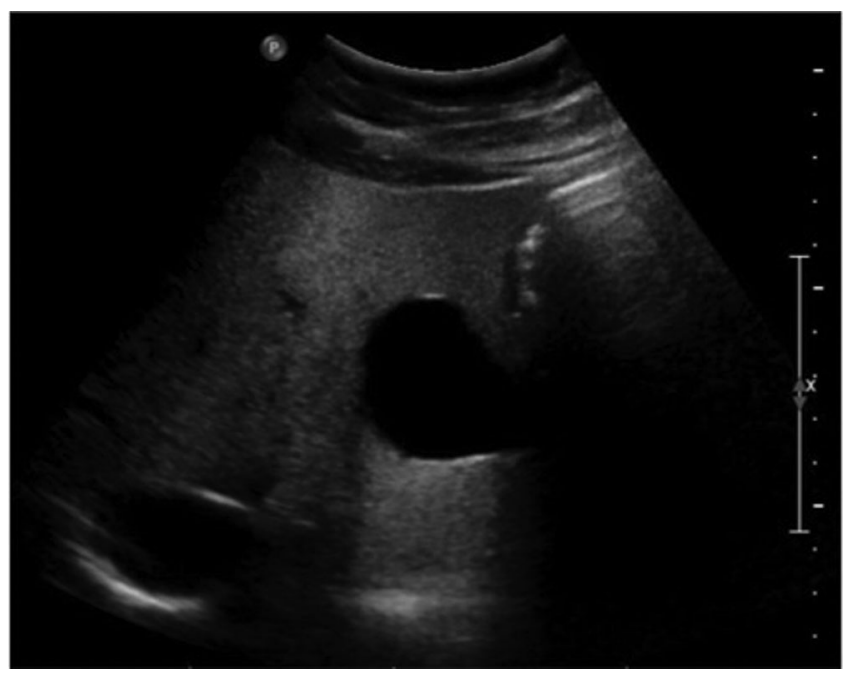

Fig. 3. Grey scale US image demonstrates an anechoic lesion in the left hepatic lobe, which has increased through transmission consistent with a simple cyst. Note the lack of septations or mural nodularity. Abbreviation: US ultrasound.

and likelihood of human error. Interobserver variability is also an issue with pathologic diagnosis. The degree of interobserver variability varies, depending on the hepatic condition. For instance, interobserver variability in the grading of hepatic fibrosis has been reflected in up to $30-50 \%$ disconcordance rates. ${ }^{40,41}$ To our knowledge, rates of interobserver variability have not been studied specifically in cystic liver disease. Sampling error is another concern with liver biopsy. Because the liver spans a large area, a biopsy can miss an area of disease. This is of particular concern with focal liver lesions. It has been reported that the diagnosis can be missed by a single pass biopsy in $20-50 \%$ of cases. ${ }^{40}$

In addition to these technical limitations, liver biopsy is an invasive procedure which carries risk of bleeding, infection, pain, or perforated viscus. However, the mortality rates of this procedure have been reported to be very low, at $0.01-$ $0.1 \% .^{40,41}$ The strengths and limitations of pathologic diagnosis are addressed in Table 4. These limitations and complications emphasize the importance of developing sensitive and specific imaging techniques to diagnose cystic liver lesions.

\section{Complications of cysts}

Complications occur most commonly in large cysts. ${ }^{3}$ Common complications include hemorrhage, rupture, infection, or biliary obstruction. Hemorrhagic cysts typically present with severe abdominal pain. On imaging, they are commonly confused with BCA or BCAC. ${ }^{42}$ Specific imaging features of hemorrhagic cysts are seen in Table 2. Cyst infection can also occur, and usually involves Gram-negative pathogens. ${ }^{43}$ It is associated with $9 \%$ mortality. ${ }^{43}$

Biliary obstruction can occur due to a mass effect of cystic lesions as they grow larger. If cysts rupture into the biliary tree, secondary cholangitis can occur. A feared complication specific to ECs is anaphylaxis, which can occur if an EC ruptures. Several other rarer complications have been reported only in case reports. Long et al. ${ }^{5}$ reports a case of a 66-year-old female with Budd-Chiari syndrome secondary to a rapidly enlarging cyst obstructing the hepatic vein. ${ }^{5}$ Another single case report described an inferior vena cava (IVC) thrombus caused by external pressure on the IVC from a simple hepatic cyst. ${ }^{44}$

\section{Management}

Asymptomatic simple cysts do not require intervention. Treatment may be indicated for those that become symptomatic. There are a number of treatment options available. Simple aspiration is not recommended as the fluid will almost always reaccumulate inside the cyst cavity. ${ }^{1}$ Fenestration or "deroofing" is a first-line surgical technique for draining a cyst, with either open or laparoscopic surgical approach. ${ }^{1,45,46}$ With this technique, the cyst is opened and drained into the peritoneum. Fenestration shows symptom reduction in $92 \%$ of cases. ${ }^{47}$ A pilot study by Wang et al. ${ }^{46}$ proved that fenestration can also be performed endoscopically, using a transgastric approach. This approach provides a minimally invasive technique and eliminates external excisions. However, access may be limited by location, especially with deeper, right-sided cysts. For instance, one of the four cases with a cyst in segment VIII required laparoscopic assistance to complete the procedure. ${ }^{46}$ Additionally, the procedure duration of endoscopic fenestration
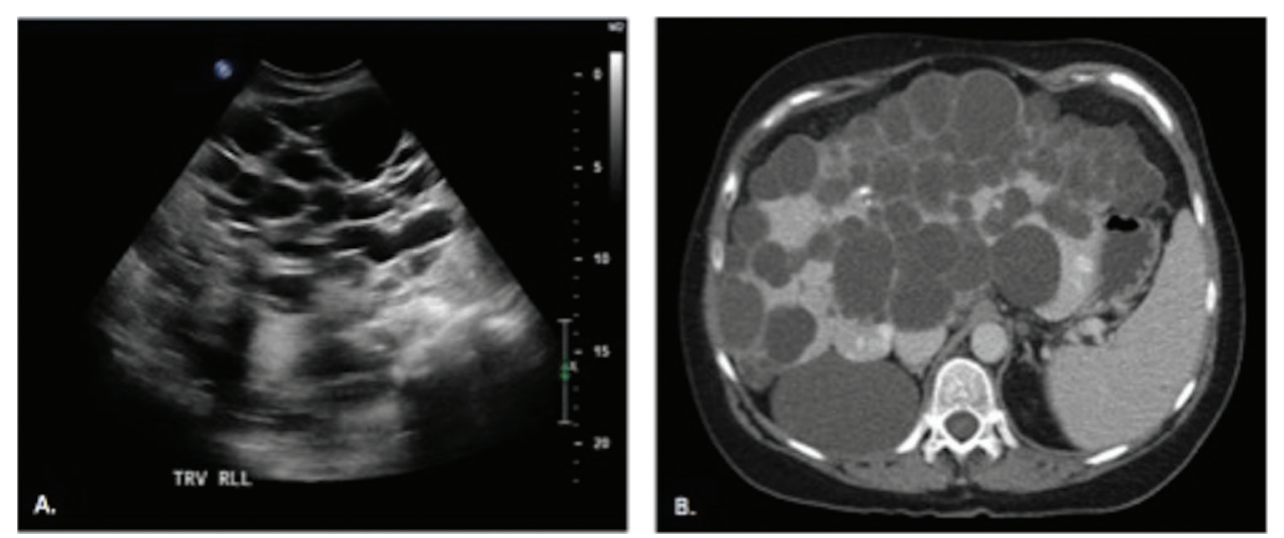

Fig. 4. (A) US image demonstrates numerous anechoic lesions occupying the majority of the right hepatic lobe, consistent with numerous hepatic cysts. (B) Contrast-enhanced CT corroborating the US findings with numerous hypodensities occupying the majority of liver consistent with polycystic liver disease. Abbreviations: CT, computed tomography; US, ultrasound. 

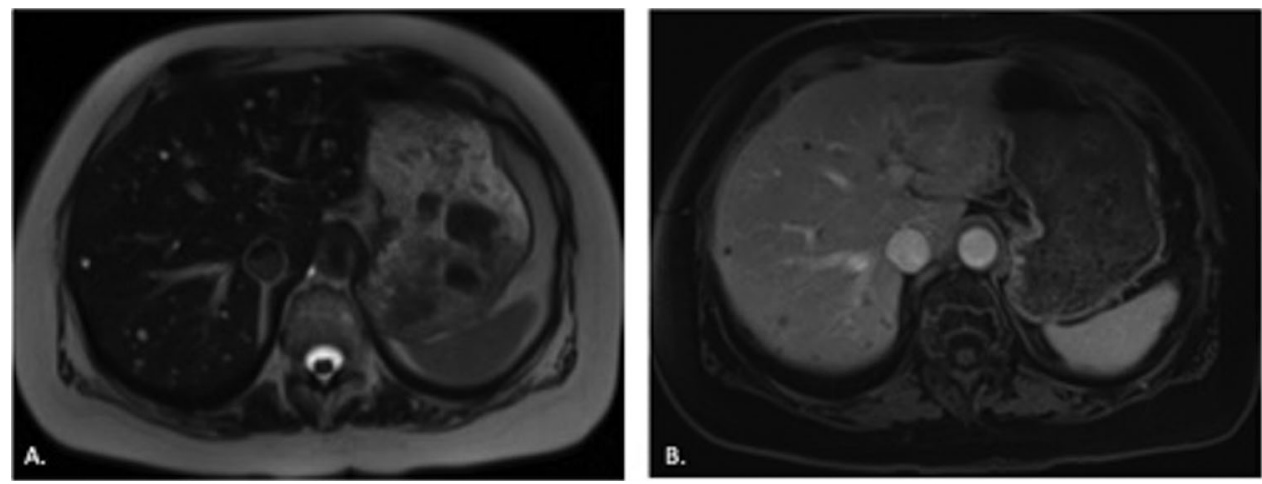

Fig. 5. A. T2-weighted MRI image demonstrates numerous subcentimeter T2 hyperintense foci in the liver. B. Postcontrast T1-weighted image with fat saturation confirms the foci do not demonstrate enhancement, consistent with biliary hamartomas. Abbreviation: MRI, magnetic resonance imaging.

ranged from $90-112 \mathrm{~min}^{46}$ compared to the average of 55 min for laparoscopic cyst drainage. ${ }^{48}$ The added time may incur additional risk in the endoscopic approach. It may also be affected by operator inexperience.

Sclerotherapy is an alternative treatment option. A sclerosing agent, such as dehydrated alcohol or a tetracycline, is injected into the cystic cavity after complete aspiration of the contents. ${ }^{6}$ It has been well documented as a simple and safe procedure. A multicenter study of 86 patients with $\mathrm{HC}$ greater than $5 \mathrm{~cm}$ found aspiration sclerotherapy to be highly efficacious. They reported reduction or complete resolution of symptoms in $89.6 \%$ of cases and $98 \%$ reduction in cyst volume when followed over a 6-month period. ${ }^{45}$ Some would argue that follow-up should take place over a longer period of time. However, cyst recurrence after aspiration typically occurs rapidly following the procedure. Other studies have shown comparable results, with $70-98 \%$ reduction in cyst size after 12-24 months. ${ }^{47,49-51}$ Interestingly, this study achieved good results after a single injection with sclerosing agent. Some suggest that multiple treatments may be required to obliterate the lesion. ${ }^{6} \mathrm{~A}$ lesser response to sclerotherapy may be seen with hemorrhagic cysts or complex cysts with large amount of intracystic debris. ${ }^{47}$
Management of complex cysts begins with specific diagnosis. As described above, imaging can be used to narrow the differential and, in some cases, may diagnose the lesion. If malignancy is suspected, a tissue sample is needed to confirm the diagnosis. In these cases, surgical resection may be indicated. A proposed algorithm for diagnosis and management is depicted in Fig. 6.

ECs should be managed with a tailored strategy (Fig. 6). Small cysts, less than $5 \mathrm{~cm}$ in diameter can be managed medically with antihelminthics and clinical observation. This is considered a "watch and wait" strategy. ${ }^{52}$ Long-term follow-up with US is indicated. However, the currently available literature does not specify a clear surveillance interval. In a retrospective review conducted over a 6-12 month period, sonographic surveillance on 47 patients with inactive ECs was performed in an effort to evaluate the "watch and wait" strategy. ${ }^{52}$ They concluded that $97.4 \%$ of cysts remained inactive. ${ }^{52}$ This study aimed to review "long-term" follow-up. However, patients were only followed over a 2-year period. More data are needed to understand the outcomes of "watch and wait" over a longer follow-up period.

If conservative therapy is unsuccessful, either percutaneous or surgical drainage is indicated. ${ }^{52}$ Definitive management

Table 4. Strengths of various diagnostic modalities for cystic liver disease

\begin{tabular}{|c|c|c|c|}
\hline Diagnostic modality & Sensitivity/Specificity, \% & Strengths & Limitations \\
\hline US & $90 / 90^{3}$ & $\begin{array}{l}\text { Inexpensive } \\
\text { Widely available } \\
\text { - No radiation exposure }\end{array}$ & $\begin{array}{l}\text { - Noncontrast } \\
\text { - Limited evaluation of } \\
\text { complex features }\end{array}$ \\
\hline CT & $>90 />70^{3,23,27}$ & $\begin{array}{l}\text { Widely available } \\
\text { Contrast enhancement can provide more } \\
\text { information }\end{array}$ & $\begin{array}{l}\text { Radiation exposure } \\
\text { Complications from } \\
\text { contrast agents }\end{array}$ \\
\hline MRI & $90-100 / 95-100^{3}$ & $\begin{array}{l}\text { No radiation exposure } \\
\text { Contrast enhancement can provide more } \\
\text { information }\end{array}$ & Expensive \\
\hline CEUS & $79-95 / 83-88^{33,36}$ & $\begin{array}{l}\text { No radiation exposure } \\
\text {. Real time imaging } \\
\text { Contrast enhancement can provide more } \\
\text { information }\end{array}$ & $\begin{array}{l}\text { Limited availability, } \\
\text { particularly in the US }\end{array}$ \\
\hline $\begin{array}{l}\text { Pathologic } \\
\text { diagnosis }\end{array}$ & Unknown & - Ability to accurately detect malignant cells & $\begin{array}{l}\text { Invasive } \\
\text { Sampling error } \\
\text { - Interobserver variability }\end{array}$ \\
\hline
\end{tabular}

Abbreviations: CEUS, contrast-enhanced ultrasound; CT, computed tomography; MRI, magnetic resonance imaging; US, ultrasound. 


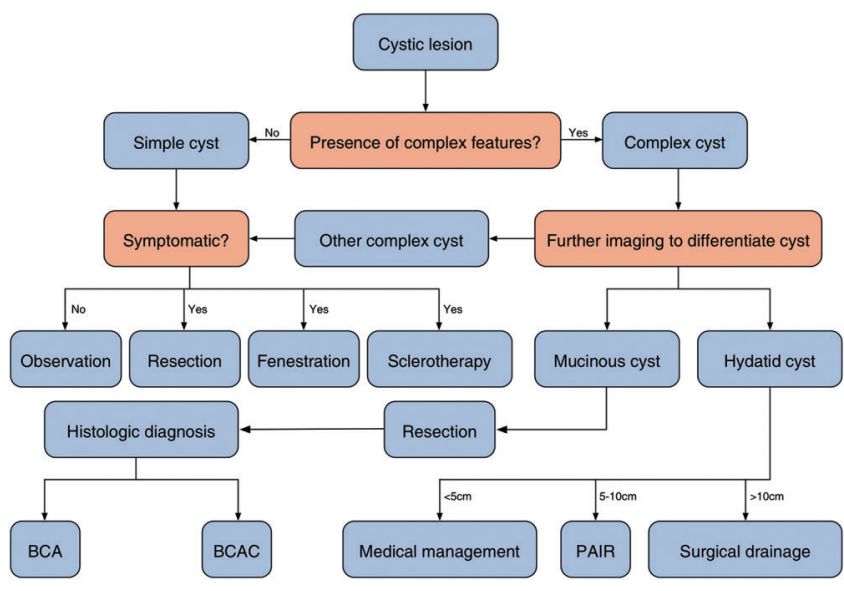

Fig. 6. Diagnostic and management strategies.

is open surgical drainage. ${ }^{23}$ However, this is an invasive option. The PAIR technique is a percutaneous treatment, which stands for "puncture, aspiration, injection, reaspiration". ${ }^{1,3}$ PAIR is indicated for ECs greater than $5-10 \mathrm{~cm}$ in diameter in patients who are poor surgical candidates or who have failed surgical intervention. ${ }^{1,27}$ In addition to drainage, antihelminthic therapy with albendazole or mebendazole is indicated. ${ }^{27}$

\section{Conclusions}

In the case of cystic liver disease, imaging can be a powerful diagnostic tool, allowing analysis of HCs in great detail. Imaging allows for early detection of potentially dangerous lesions, such as BCAC, EC, and cystic HCC. But, it also has the potential for invasive, costly, and often unnecessary work-ups with detection of asymptomatic incidentalomas.

The primary goals in evaluation and treatment of HCs are to identify any malignant or premalignant lesions, to treat any symptoms associated with $\mathrm{HCs}$, and to prevent complications for HC. Although the majority of HCs are benign, there are several malignant (HCC and BCAC) and premalignant (BCA and IDPN). Mortality is increased in malignant cysts and those with complications. Therefore, it is important to accurately diagnose and treat suspicious cysts to prevent these situations of increased risk.

\section{Acknowledgements}

This work was made possible by the Herman Lopata Chair in Hepatitis Research, and a grant from Alexion Corp. We are also grateful to Peg Atkinson Lee for assistance in the production of the original graphics included in this publication.

\section{Conflict of interest}

The authors have no conflict of interests related to this publication.

\section{Author contributions}

Drafting the manuscript and development of figures (MGM), providing background information and outline for the draft
(TP), analysis of imaging for figures and revisions (MM), proposing concept for review and revising the manuscript with critical revisions (GYW).

\section{References}

[1] Marrero JA, Ahn J, Rajender Reddy K. ACG clinical guideline: the diagnosis and management of focal liver lesions. Am J Gastroenterol 2014;109:13281347. doi: 10.1038 /ajg.2014.213.

[2] Sanfelippo PM, Beahrs OH, Weiland LH. Cystic disease of the liver. Ann Surg 1974;179:922-925. doi: 10.1097/00000658-197406000-00018.

[3] Lantinga MA, Gevers TJ, Drenth JP. Evaluation of hepatic cystic lesions. World J Gastroenterol 2013;19:3543-3554. doi: 10.3748/wjg.v19.i23.3543.

[4] Inan N, Arslan A, Akansel G, Anik Y, Sarisoy HT, Ciftci E, et al. Diffusionweighted imaging in the differential diagnosis of simple and hydatid cysts of the liver. AJR Am J Roentgenol 2007;189:1031-1036. doi: 10.2214/AJR. 07.2251

[5] Long J, Vaughan-Williams H, Moorhouse J, Sethi H, Kumar N. Acute BuddChiari syndrome due to a simple liver cyst. Ann R Coll Surg Engl 2014;96: 109E-111E. doi: 10.1308/003588414X13824511649698.

[6] Karam AR, Connolly C, Fulwadhva U, Hussain S. Alcohol sclerosis of a giant liver cyst following failed deroofings. J Radiol Case Rep 2011;5:19-22. doi: $10.3941 /$ jrcr.v5i2.634.

[7] Wang C, Miao R, Liu H, Du X, Liu L, Lu X, et al. Intrahepatic biliary cystadenoma and cystadenocarcinoma: an experience of 30 cases. Dig Liver Dis 2012;44:426-431. doi: 10.1016/j.dld.2011.11.007.

[8] Horsmans Y, Laka A, Gigot JF, Geubel AP. Serum and cystic fluid CA 19-9 determinations as a diagnostic help in liver cysts of uncertain nature. Liver 1996;16:255-257. doi: 10.1111/j.1600-0676.1996.tb00738.x.

[9] Lee JH, Chen DR, Pang SC, Lai YS. Mucinous biliary cystadenoma with mesenchymal stroma: expressions of CA 19-9 and carcinoembryonic antigen in serum and cystic fluid. J Gastroenterol 1996;31:732-736. doi: 10.1007/ BF02347626.

[10] Zhang FB, Zhang AM, Zhang ZB, Huang X, Wang XT, Dong JH. Preoperative differential diagnosis between intrahepatic biliary cystadenoma and cystadenocarcinoma: a single-center experience. World J Gastroenterol 2014;20: 12595-12601. doi: 10.3748/wjg.v20.i35.12595.

[11] Yanai H, Tada N. A simple hepatic cyst with elevated serum and cyst fluid CA19-9 levels: a case report. J Med Case Rep 2008;2:329. doi: 10.1186/ 1752-1947-2-329.

[12] Vachha B, Sun MR, Siewert B, Eisenberg RL. Cystic lesions of the liver. AJR Am J Roentgenol 2011;196:W355-W366. doi: 10.2214/AJR.10.5292.

[13] Bakoyiannis A, Delis S, Triantopoulou C, Dervenis C. Rare cystic liver lesions: a diagnostic and managing challenge. World J Gastroenterol 2013;19:76037619. doi: $10.3748 /$ wjg.v19.i43.7603.

[14] Abu-Wasel B, Walsh C, Keough V, Molinari M. Pathophysiology, epidemiology, classification and treatment options for polycystic liver diseases. World J Gastroenterol 2013;19:5775-5786. doi: 10.3748/wjg.v19.i35.5775.

[15] Qian LJ, Zhu J, Zhuang ZG, Xia Q, Liu Q, Xu JR. Spectrum of multilocular cystic hepatic lesions: CT and MR imaging findings with pathologic correlation. Radiographics 2013;33:1419-1433. doi: 10.1148/rg.335125063.

[16] Dong Y, Wang WP, Mao F, Fan M, Ignee A, Serra C, et al. Contrast enhanced ultrasound features of hepatic cystadenoma and hepatic cystadenocarcinoma. Scand J Gastroenterol 2017;52:365-372. doi: 10.1080/00365521. 2016.1259652.

[17] van Seventer I, Verheij J, Phoa S, van Gulik TM. A cystic, septated lesion in the liver with unusual diagnosis. BMJ Case Rep 2014;2014. doi: 10.1136/ bcr-2014-207687.

[18] Corvino A, Catalano O, Setola SV, Sandomenico F, Corvino F, Petrillo A. Contrast-enhanced ultrasound in the characterization of complex cystic focal liver lesions. Ultrasound Med Biol 2015;41:1301-1310. doi: 10.1016/j. ultrasmedbio.2014.12.667.

[19] Xu HX, Lu MD, Liu LN, Zhang YF, Guo LH, Liu C, et al. Imaging features of intrahepatic biliary cystadenoma and cystadenocarcinoma on B-mode and contrast-enhanced ultrasound. Ultraschall Med 2012;33:E241-E249. doi: 10.1055/s-0031-1299276

[20] Martie A, Bota S, Sporea I, Sirli R, Popescu A, Danila M. The contribution of contrast enhanced ultrasound for the characterization of benign liver lesions in clinical practice - a monocentric experience. Med Ultrason 2012;14:283-287.

[21] Watanabe A, Suzuki H, Kubo N, Araki K, Kobayashi T, Sasaki S, et al. An oncocytic variant of intraductal papillary neoplasm of the bile duct that formed a giant hepatic cyst. Rare Tumors 2013;5:e30. doi: 10.4081/rt. 2013.e30.

[22] Kim HJ, Yu ES, Byun JH, Hong SM, Kim KW, Lee JS, et al. CT differentiation of mucin-producing cystic neoplasms of the liver from solitary bile duct cysts. AJR Am J Roentgenol 2014;202:83-91. doi: 10.2214/AJR.12.9170.

[23] Pakala T, Molina M, Wu GY. Hepatic echinococcal cysts: a review. J Clin Transl Hepatol 2016;4:39-46. doi: 10.14218/JCTH.2015.00036. 
[24] Hsu M, Terris B, Wu TT, Zen Y, Eng HL, Huang WT, et al. Endometrial cysts within the liver: a rare entity and its differential diagnosis with mucinous cystic neoplasms of the liver. Hum Pathol 2014;45:761-777. doi: 10.1016/ j.humpath.2013.11.005.

[25] Huang WT, Chen WJ, Chen CL, Cheng YF, Wang JH, Eng HL. Endometrial cyst of the liver: a case report and review of the literature. J Clin Pathol 2002;55: 715-717. doi: 10.1136/jcp.55.9.715.

[26] Poddar U, Chawla Y, Dilawari JB, Kataria RN, Behera A, Suri SU. Post-biopsy liver cyst: a rare complication of liver biopsy. J Gastroenterol Hepatol 1999; 14:97-99. doi: 10.1046/j.1440-1746.1999.01797.x.

[27] Scherer K, Gupta N, Caine WP, Panda M. Differential diagnosis and management of a recurrent hepatic cyst: a case report and review of literature. J Gen Intern Med 2009;24:1161-1165. doi: 10.1007/s11606-009-1062-1.

[28] Monzawa S, Ichikawa T, Nakajima H, Kitanaka Y, Omata K, Araki T. Dynamic CT for detecting small hepatocellular carcinoma: usefulness of delayed phase imaging. AJR Am J Roentgenol 2007;188:147-153. doi: 10.2214/ AJR.05.0512.

[29] Miller FH, Butler RS, Hoff FL, Fitzgerald SW, Nemcek AA Jr, Gore RM. Using triphasic helical CT to detect focal hepatic lesions in patients with neoplasms. AJR Am J Roentgenol 1998;171:643-649. doi: 10.2214/ajr.171.3.9725290.

[30] Erturk SM, Ichikawa T, Kaya E, Yapici O, Ozel A, Mahmutoglu AS, et al. Diffusion tensor imaging of cysts, hemangiomas, and metastases of the liver. Acta Radiol 2014;55:654-660. doi: 10.1177/0284185113504916.

[31] Oruç E, Yõldõrõm N, Topal NB, Kõlõçturgay S, Akgöz S, Savcõ G. The role of diffusion-weighted MRI in the classification of liver hydatid cysts and differentiation of simple cysts and abscesses from hydatid cysts. Diagn Interv Radiol 2010;16:279-287. doi: 10.4261/1305-3825.DIR.2807-09.2.

[32] D'Onofrio M, Crosara S, De Robertis R, Canestrini S, Mucelli RP. Contrastenhanced ultrasound of focal liver lesions. AJR Am J Roentgenol 2015;205: W56-W66. doi: 10.2214/AJR.14.14203.

[33] Strobel D, Seitz K, Blank W, Schuler A, Dietrich C, von Herbay A, et al. Contrast-enhanced ultrasound for the characterization of focal liver lesions-diagnostic accuracy in clinical practice (DEGUM multicenter trial). Ultraschall Med 2008;29:499-505. doi: 10.1055/s-2008-1027806.

[34] Chung YE, Kim KW. Contrast-enhanced ultrasonography: advance and current status in abdominal imaging. Ultrasonography 2015;34:3-18. doi: $10.14366 /$ usg. 14034 .

[35] Kong WT, Wang WP, Huang BJ, Ding H, Mao F. Value of wash-in and wash-out time in the diagnosis between hepatocellular carcinoma and other hepatic nodules with similar vascular pattern on contrast-enhanced ultrasound. J Gastroenterol Hepatol 2014;29:576-580. doi: 10.1111/jgh.12394.

[36] Tranquart F, Le Gouge A, Correas JM, Ladam Marcus V, Manzoni P, Vilgrain V, et al. Role of contrast-enhanced ultrasound in the blinded assessment of focal liver lesions in comparison with MDCT and CEMRI: Results from a multicentre clinical trial. European Journal of Cancer Supplements 2008;6:9-15. doi: $10.1016 /$ j.ejcsup.2008.06.003.

[37] Bernatik T, Schuler A, Kunze G, Mauch M, Dietrich CF, Dirks K, et al. Benefit of contrast-enhanced ultrasound (CEUS) in the follow-up care of patients with colon cancer: a prospective multicenter study. Ultraschall Med 2015;36: 590-593. doi: 10.1055/s-0041-107833.
[38] Correas JM, Low G, Needleman L, Robbin ML, Cosgrove D, Sidhu PS, et al. Contrast enhanced ultrasound in the detection of liver metastases: a prospective multi-centre dose testing study using a perfluorobutane microbubble contrast agent (NC100100). Eur Radiol 2011;21:1739-1746. doi: 10. 1007/s00330-011-2114-6.

[39] Berdel HO, Maxey M, Smith RS. A simple hepatic cyst masquerading as a complex cyst. Am Surg 2014;80:E255-E256.

[40] Sanai FM, Keeffe EB. Liver biopsy for histological assessment: The case against. Saudi J Gastroenterol 2010;16:124-132. doi: 10.4103/13193767.61244.

[41] Rockey DC, Caldwell SH, Goodman ZD, Nelson RC, Smith AD. Liver biopsy. Hepatology 2009;49:1017-1044. doi: 10.1002/hep.22742.

[42] Tonolini M, Crespi M, Bianco R. Magnetic resonance imaging clinches diagnosis of haemorrhagic liver cyst. Dig Liver Dis 2013;45:1061. doi: 10 1016/j.dld.2013.05.012.

[43] Lantinga MA, Geudens A, Gevers TJ, Drenth JP. Systematic review: the management of hepatic cyst infection. Aliment Pharmacol Ther 2015;41:253261. doi: 10.1111/apt.13047.

[44] Musielak MC, Singh R, Hartman E, Bernstein J. Simple hepatic cyst causing inferior vena cava thrombus. Int J Surg Case Rep 2014;5:339-341. doi: 10. 1016/j.ijscr.2014.03.009.

[45] Hansen P, Bhoyrul S, Legha P, Wetter A, Way LW. Laparoscopic treatment of liver cysts. J Gastrointest Surg 1997;1:53-59. doi: 10.1007/s11605-0060010-1.

[46] Wang D, Liu Y, Chen D, Li X, Wu R, Liu W, et al. Flexible transgastric endoscopic liver cyst fenestration: A feasibility study in humans (with video). Medicine (Baltimore) 2016;95:e5420. doi: 10.1097/MD.0000000000005420.

[47] Wijnands TF, Ronot M, Gevers TJ, Benzimra J, Kool LJ, Vilgrain V, et al. Predictors of treatment response following aspiration sclerotherapy of hepatic cysts: an international pooled analysis of individual patient data. Eur Radiol 2017;27:741-748. doi: 10.1007/s00330-016-4363-x.

[48] Neri V, Ambrosi A, Fersini A, Valentino TP. Laparoscopic treatment of biliary hepatic cysts: short- and medium-term results. HPB (Oxford) 2006;8:306310. doi: 10.1080/13651820500465766.

[49] Yang CF, Liang HL, Pan HB, Lin YH, Mok KT, Lo GH, et al. Single-session prolonged alcohol-retention sclerotherapy for large hepatic cysts. AJR Am J Roentgenol 2006;187:940-943. doi: 10.2214/AJR.05.0621.

[50] Yu JH, Du Y, Li Y, Yang HF, Xu XX, Zheng HJ, et al. Effectiveness of CT-guided sclerotherapy with estimated ethanol concentration for treatment of symptomatic simple hepatic cysts. Clin Res Hepatol Gastroenterol 2014;38:190194. doi: 10.1016/j.clinre.2013.09.008.

[51] Montorsi M, Torzilli G, Fumagalli U, Bona S, Rostai R, De Simone M, et al. Percutaneous alcohol sclerotherapy of simple hepatic cysts. Results from a multicentre survey in Italy. HPB Surg 1994;8:89-94. doi: 10.1155/1994/10372.

[52] Piccoli L, Tamarozzi F, Cattaneo F, Mariconti M, Filice C, Bruno A, et al. Longterm sonographic and serological follow-up of inactive echinococcal cysts of the liver: hints for a "watch-and-wait" approach. PLoS Negl Trop Dis 2014;8: e3057. doi: 10.1371/journal.pntd.0003057. 